\title{
Ray-path concepts for converted-wave seismic refraction
}

\author{
Steve Hearn ${ }^{1,2}$ Alan Meulenbroek ${ }^{1}$ \\ ${ }^{1}$ Velseis Pty Ltd and School of Earth Sciences, University of Queensland, PO Box 118, Sumner Park, \\ QLD 4074, Australia. \\ ${ }^{2}$ Corresponding author. Email: steveh@velseis.com
}

\begin{abstract}
P-wave reflection-statics solutions typically incorporate $\mathrm{P}$-wave refraction data, derived from the first breaks of the production data. Similarly, converted-wave refractions, taken from inline-component recordings, can be exploited to yield $\mathrm{S}$-wave receiver statics, required in the processing of converted-wave reflection data. This methodology requires extensions to well known P-wave refraction analysis methods. This paper outlines extensions of the slope-intercept method and the reciprocal method, required to analyse converted-wave refractions. We discuss the computation of S-wave time-depths and describe how the observed ratio of $\mathrm{S}$-wave to $\mathrm{P}$-wave time-depths can provide a useful estimate of the near-surface $V_{\mathrm{P}} / V_{\mathrm{S}}$ ratio, which is of interest in the analysis of engineering rock strengths.

We also include discussion of several related practical issues, with particular reference to dynamite sources. When the source is buried in the refractor, the required reciprocal times cannot be directly measured from the raw travel-time data. They can, however, be easily derived via correction using measured intercept times. Often converted-wave refractions are of poorer quality than conventional P-wave refractions, such that reversed refractions may not be available over some parts of the spread. In this situation, the preferred time-depth quantity cannot be computed. However, delay-times derived from singleended data can be substituted, particularly if lateral variations in refractor velocity are allowed for.

The concepts outlined here are used in a companion paper to correct $\mathrm{S}$-wave receiver statics in a coal-scale dataset from the Bowen Basin in central Queensland.
\end{abstract}

Key words: converted-wave, delay-time, reciprocal method, seismic refraction, slope-intercept.

\section{Introduction}

The basic theory and methodology of compressional-wave (P-wave) seismic refraction have been known for many years, and are well documented (e.g. Musgrave, 1967; Palmer, 1986). First-arrivals on P-wave reflection records are routinely analysed to extract refraction static solutions. The past decade has seen renewed commercial interest in shear-wave ( $\mathrm{S}$-wave) exploration, with converted-wave reflection (using a P-wave source) now being the preferred methodology for both onshore and offshore surveys. A significant aspect of onshore convertedwave processing relates to the challenging issue of receiver S-wave statics.

This paper serves several purposes. First, it reviews aspects of conventional refraction theory. This provides the basis for several simple extensions required for converted-wave statics use. As part of this discussion, several practical implications are noted. Real data examples are given in a companion paper (Meulenbroek and Hearn, 2011).

\section{Mode-converted refraction: terminology and geometry}

We consider here the simple (but practically useful) two-layer, isotropic, refraction model. We will initially restrict ourselves to planar interfaces, and then relax this assumption. In the context of converted-wave reflection we assume a P-wave source. In general, a refraction ray path comprises three sections: from source down to refractor, along the refractor, and the head wave from refractor to surface. In conventional refraction these components are all P-waves, and we will describe this event as a PPP refraction. Due to mode conversion, other phases such as PPS, PSP and PSS can occur subject to appropriate layer properties. The PPP and PPS phases are of most practical interest since they occur as the first major arrivals on the vertical and inline-horizontal components respectively. We concentrate on these phases here.

As is the case with conventional refraction, the orientation of a converted-refraction is governed by Snell's Law. As indicated in Figure 1, the PPP and PPS refractions are identical in the source region, with a critical angle $\left(i_{\mathrm{P}}\right)$ governed by the $\mathrm{P}$-wave velocities in the overburden and refractor ( $V_{1 \mathrm{P}}$ and $V_{2 \mathrm{P}}$ respectively):

$$
i_{\mathrm{P}}=\sin ^{-1}\left(V_{1 \mathrm{P}} / V_{2 \mathrm{P}}\right)
$$

In the receiver region, the PPP refraction has the same critical angle $\left(i_{\mathrm{P}}\right)$, while the critical angle for the upgoing PPS headwave $\left(i_{S}\right)$ is influenced by the S-wave velocity in the overburden $\left(V_{1 \mathrm{~S}}\right)$, according to:

$$
i_{S}=\sin ^{-1}\left(V_{1 \mathrm{~S}} / V_{2 \mathrm{P}}\right)
$$

\section{Slope-intercept interpretation for PPP refraction}

This most basic form of interpretation, appropriate for planar interfaces, is based on analysis of linear segments on a travel-time versus distance plot. The technique is well known (e.g. Nettleton, 1940; Knox, 1967; Palmer, 1986). Here we include a concise overview, for the two-layer case, as a prelude to the convertedwave extension. Figure $2 a$ shows PPP refraction paths from Shots A and B into a Geophone G, for the case of a dipping, planar refractor. The corresponding travel-time versus distance graph is shown in Figure $2 b$. Segments for the direct P-waves and the PPP refractions are shown.

Typically, an estimate of the overburden P-wave velocity $\left(V_{1 \mathrm{P}}\right)$ in the vicinity of a shot point is derived from the slope 


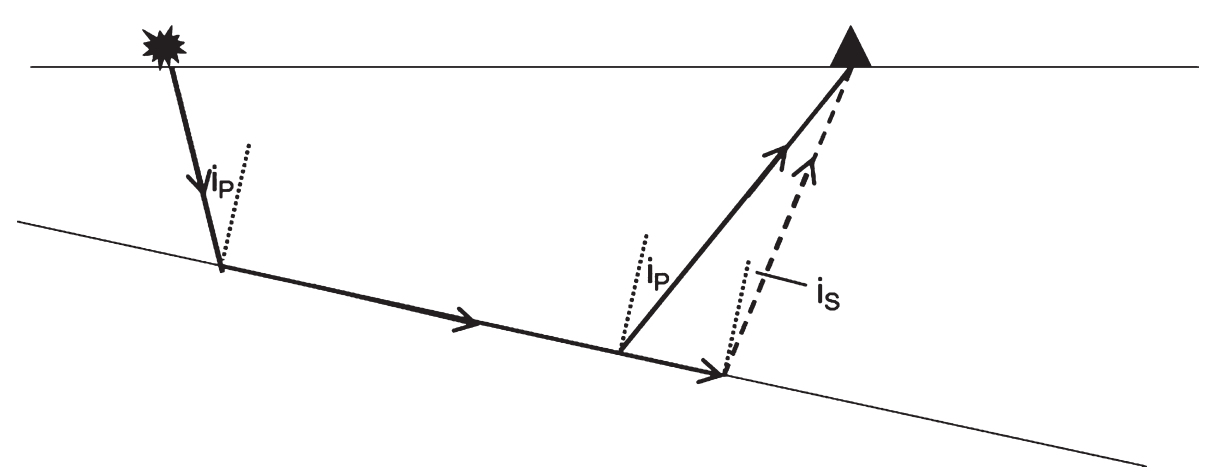

Fig. 1. PPP and PPS refraction paths. Here, and in subsequent figures, $\mathrm{P}$ - and S-wave types are represented by solid and dashed rays, respectively. As outlined in the text, the critical angle for the PPP refraction $\left(i_{\mathrm{P}}\right)$ is identical in the source and receiver regions, while the critical angle for the PPS head-wave $\left(i_{\mathrm{S}}\right)$ is influenced by the S-wave velocity in the overburden.

(a)
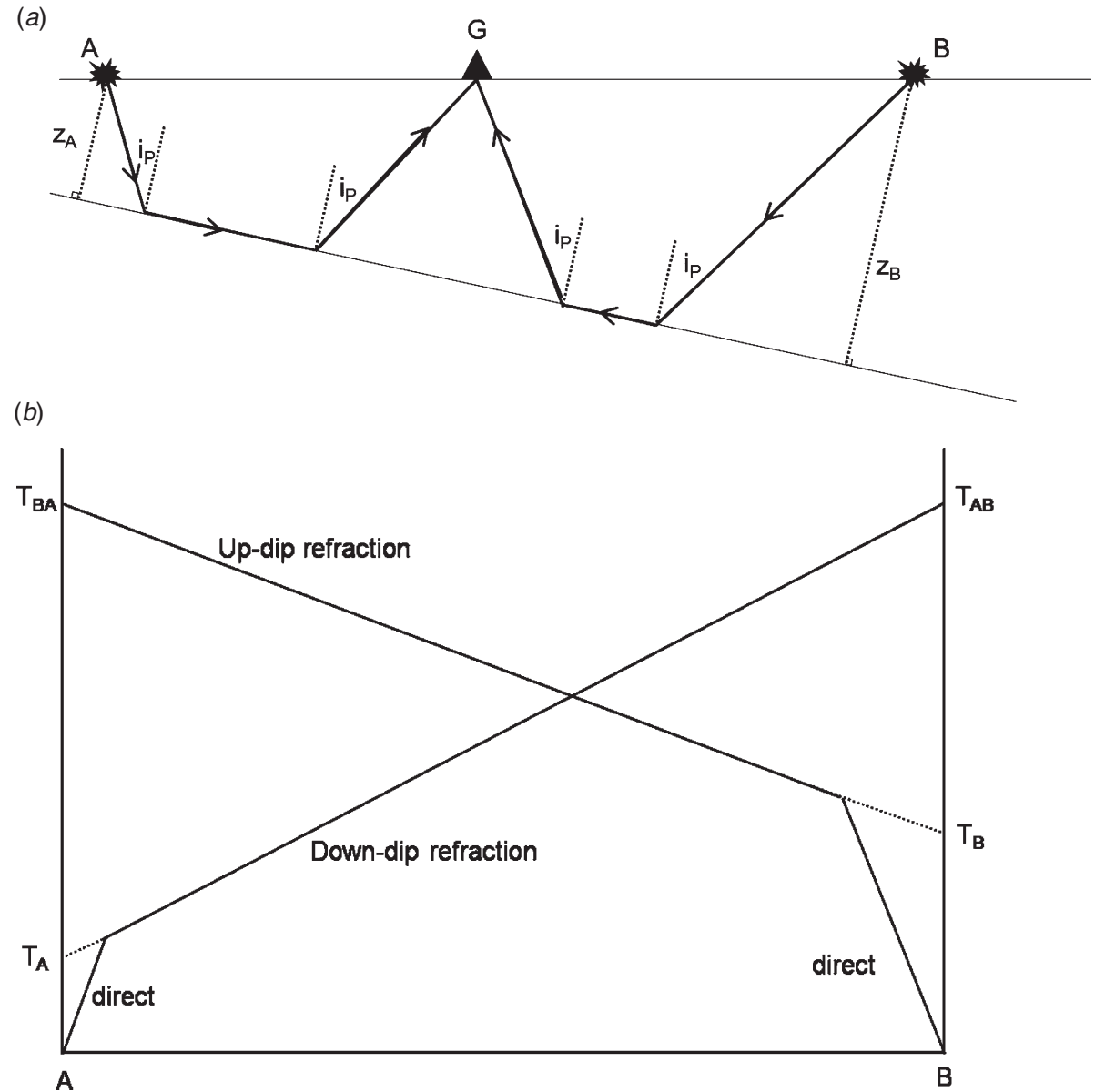

Fig. 2. Slope-intercept PPP interpretation for a dipping layer model. (a) Down-dip and up-dip PPP refraction paths from Shots A and B into Geophone G. Critical angle $\left(i_{\mathrm{P}}\right)$ and perpendicular depths $\left(Z_{\mathrm{A}}, Z_{\mathrm{B}}\right)$ are shown. (b) Corresponding time versus distance curves, including direct and refracted segments. Intercept times $\left(T_{\mathrm{A}}, T_{\mathrm{B}}\right)$ and reciprocal times $\left(T_{\mathrm{AB}}, T_{\mathrm{BA}}\right)$ are shown.

of the direct-arrival segment on the time versus distance plot. The slope of the refraction segment is affected by both the dip and velocity of the refractor. This ambiguity can be overcome if reversed refraction data are recorded. As seen in Figure $2 b$, the slope is increased in the down-dip direction, and reduced in the up-dip direction. The refractor velocity can be estimated as:

$$
V_{2 \mathrm{P}} \approx 1 / \text { (average slope of down-dip and up-dip refraction }
$$
segments).
The intercept of the PPP refraction branch at a shotpoint is related to the perpendicular depth to the refractor at that shotpoint. For example, at Shot A, the intercept time is

$$
T_{\mathrm{A}}=2 Z_{\mathrm{A}} \cos i_{\mathrm{P}} / V_{1 \mathrm{P}} .
$$

Since $V_{1 \mathrm{P}}$ and $V_{2 \mathrm{P}}$ have been estimated using slopes, the critical angle $i_{\mathrm{P}}$ can be obtained using equation 1 . Equation 4 can then be used to derive the depth $Z_{\mathrm{A}}$ from the measured intercept time at the shotpoint $\left(T_{\mathrm{A}}\right)$. 
Finally note that because of ray-path reciprocity, the total refraction time from a shot at $\mathrm{A}$ to a receiver at $\mathrm{B}\left(T_{\mathrm{AB}}\right)$ must equal the refraction time from $\mathrm{B}$ to $\mathrm{A}\left(T_{\mathrm{BA}}\right)$. This observation forms the basis for a range of reversed-spread interpretation techniques discussed below. Following Hawkins (1961) we will use the term reciprocal-time for this quantity.

\section{Slope-intercept interpretation for PPS refraction}

Figure $3 a$ shows PPS refraction paths from Shots A and B into a Geophone G, again for the general case of a dipping refractor. Figure $3 b$ shows the corresponding travel-time versus distance graph for these PPS refractions. For reference, the segments for the direct P-waves and the PPP refractions are repeated from Figure $2 b$.

Note that in the absence of dip, the PPP and PPS refraction curves would be parallel, since they have the same wave type in the refractor. However, Figure $3 b$ illustrates that the PPS refraction is more strongly affected by dip than PPP. Dip affects the slope because the head-wave path length is changing laterally, and this effect is amplified for the lowervelocity S head waves. Hence the PPP and PPS branches are no longer parallel.
As noted above, the refractor velocity can be estimated by averaging the slopes on the PPP segments (equation 3). An alternative estimate could, in theory, be obtained by applying equation 3 to the down-dip and up-dip PPS refraction segments. In practice, however, the PPP estimate is preferred, because dip has less influence, and because the PPP arrivals are generally of higher quality (e.g. Meulenbroek and Hearn, 2011).

The intercept for the PPS refraction is influenced by both the $\mathrm{P}$-wave and $\mathrm{S}$-wave velocities in the surface layer. Hence, compared with equation 4, the PPS intercept at Shot A can be shown easily to have two terms:

$$
T_{\mathrm{A}}{ }^{\prime}=Z_{\mathrm{A}}\left(\cos i_{\mathrm{P}} / V_{1 \mathrm{P}}+\cos i_{\mathrm{S}} / V_{1 \mathrm{~S}}\right)
$$

As will be explained below, this intercept represents the sum of $\mathrm{P}$-wave and $\mathrm{S}$-wave time depths at the shot point. This expression could, in theory, be inverted to obtain the depth $Z_{\mathrm{A}}$, provided estimates of both $V_{1 \mathrm{P}}$ and $V_{1 \mathrm{~S}}$ were available. In practice, it is usually easier to obtain $Z_{\mathrm{A}}$ from the PPP refractions, using equation 4.

We noted above that, for PPP refractions, the so-called reciprocal times $\left(T_{\mathrm{AB}}\right.$ and $\left.T_{\mathrm{BA}}\right)$ are equal. However, as

(a)
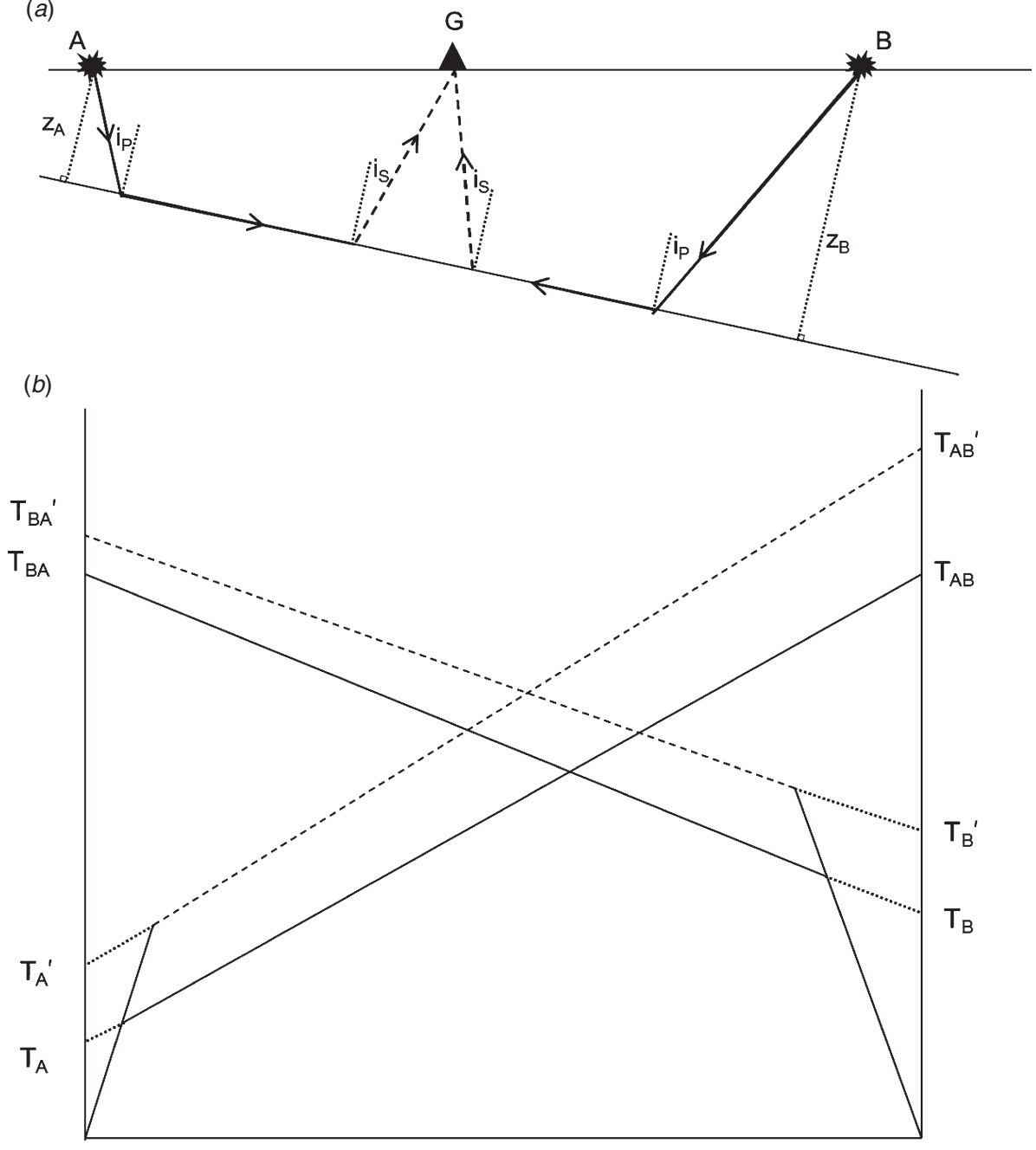

A

B

Fig. 3. Slope-intercept interpretation for PPS converted refractions on a dipping layer model. (a) Down-dip and up-dip PPS refraction paths from Shots A and B into Geophone G. The S head-wave segments are shown dashed. (b) Time versus distance curves for the PPS refractions are added to the PPP curves from Figure $2 b$. The segment $T_{\mathrm{A}}{ }^{\prime} \rightarrow T_{\mathrm{AB}}{ }^{\prime}$ is the PPS refraction segment from Shot $\mathrm{A}$. The segment $T_{\mathrm{B}}{ }^{\prime} \rightarrow T_{\mathrm{BA}^{\prime}}$ is the PPS refraction segment from Shot B. 
indicated in Figure $3 b$, the total PPS refraction time from the shot at $\mathrm{A}$ to a receiver at $\mathrm{B}\left(T_{\mathrm{AB}}{ }^{\prime}\right)$ is not equal to the $\mathrm{PPS}$ refraction time from B to A $\left(T_{\mathrm{BA}}{ }^{\prime}\right)$. Reciprocity does not apply for PPS refractions, since the ray paths are different in the forward and reverse directions (Figure 4). The breakdown of reciprocity is of practical importance in all areas of converted-wave seismology. For example, in converted-wave reflection the term diodic illumination has been coined to describe the differences seen between images constructed with positive and negative offset rays (e.g. Thompson, 1999; Li et al., 2001).

\section{Reciprocal method for PPP refraction}

When the refracting interface and/or the surface are not planar, the slope-intercept method may provide a first approximation, but a more rigorous approach to interpretation is needed. An effective technique for handling interface topography, and ambiguity between refractor dip and velocity, is to simultaneously analyse forward and reverse refraction arrivals at a geophone. Refraction-statics algorithms used in 2D reflection processing often utilise this reversed-spread approach. Variations on this theme include the method of differences (Edge and Laby, 1931), Hagiwara's method (Hagiwara and Omote, 1939), the plus-minus method (Hagedoorn, 1959), the ABC method (Nettleton, 1940), the reciprocal method (Hawkins, 1961), and the generalised reciprocal method (Palmer, 1981). Here we will follow the conventional reciprocal method. The core theory presented here is applicable to all variations of the method.

In order to overcome the inherent ambiguity between refractor velocity and dip, the reciprocal method makes use of refractions recorded at a geophone from two shots (A, B) in opposite directions from the geophone (Figure 5). At any geophone, the time-depth $\left(t_{\mathrm{G}}\right)$ is computed from measured quantities $t_{\mathrm{F}}, t_{\mathrm{R}}$, and $T_{\mathrm{AB}}$ according to:

$$
t_{\mathrm{G}}=0.5\left(t_{\mathrm{F}}+t_{\mathrm{R}}-T_{\mathrm{AB}}\right)
$$

where $t_{\mathrm{F}}$ is the refraction time in the 'forward' direction (i.e. from Shot $\mathrm{A}), t_{\mathrm{R}}$ is the refraction time in the 'reverse' direction (i.e. from Shot $\mathrm{B}$ ) and $T_{\mathrm{AB}}$ is the reciprocal time (time for a refraction from Shot $\mathrm{A}$ to a geophone at $\mathrm{B}$, or vice versa).

Consideration of equation 6 and Figure 5 indicates that subtraction of the reciprocal time $\left(T_{\mathrm{AB}}\right)$ effectively cancels segments of the forward and reverse times $\left(t_{\mathrm{F}}\right.$ and $\left.t_{\mathrm{R}}\right)$, such that the time-depth reduces to

$$
t_{\mathrm{G}}=\mathrm{DG} / V_{1 \mathrm{P}}-\mathrm{DG}^{\prime} / V_{2 \mathrm{P}}
$$

where the path lengths are as shown in Figure 5, and the velocities are as defined above. This expression allows convenient comparison to the delay-time as below. Trigonometric analysis of the residual segments in equation 7 (Hawkins, 1961) reveals that the perpendicular depth to the refractor $\left(Z_{\mathrm{G}}\right)$ can be derived from the time-depth via:

$$
Z_{\mathrm{G}}=t_{\mathrm{G}} V_{1 \mathrm{P}} / \cos i_{\mathrm{P}}
$$

where $i_{\mathrm{P}}$ is the critical angle for PPP refraction, obtained via equation 1 . Note that for this step the velocities $V_{1 \mathrm{P}}$ and $V_{2 \mathrm{P}}$ are required. The overburden velocity $\left(V_{1 \mathrm{P}}\right)$ is normally derived from the slope of the direct-arrival segment (as in the Slope-Intercept method). The refractor velocity $\left(V_{2 \mathrm{P}}\right)$ can be accurately derived using a convenient velocity function computed at each geophone. One suitable function is:

$$
t_{\mathrm{V}}=0.5\left(t_{\mathrm{F}}-t_{\mathrm{R}}+T_{\mathrm{AB}}\right)
$$

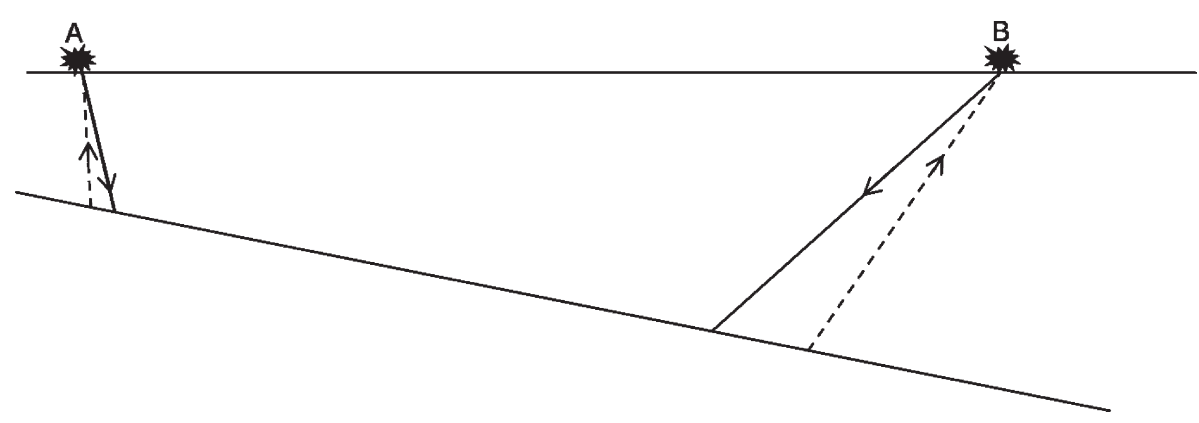

Fig. 4. PPS refraction paths from shot at $\mathrm{A}$ to receiver at $\mathrm{B}$, and from shot at $\mathrm{B}$ to receiver at $\mathrm{A}$. For the situation shown here the forward time $\left(T_{\mathrm{AB}}{ }^{\prime}\right.$ on Figure 3$)$ would normally be greater than the reverse time $\left(T_{\mathrm{BA}^{\prime}}\right)$, since the upgoing $\mathrm{S}$ head-wave path is longer. Hence reciprocity does not apply.

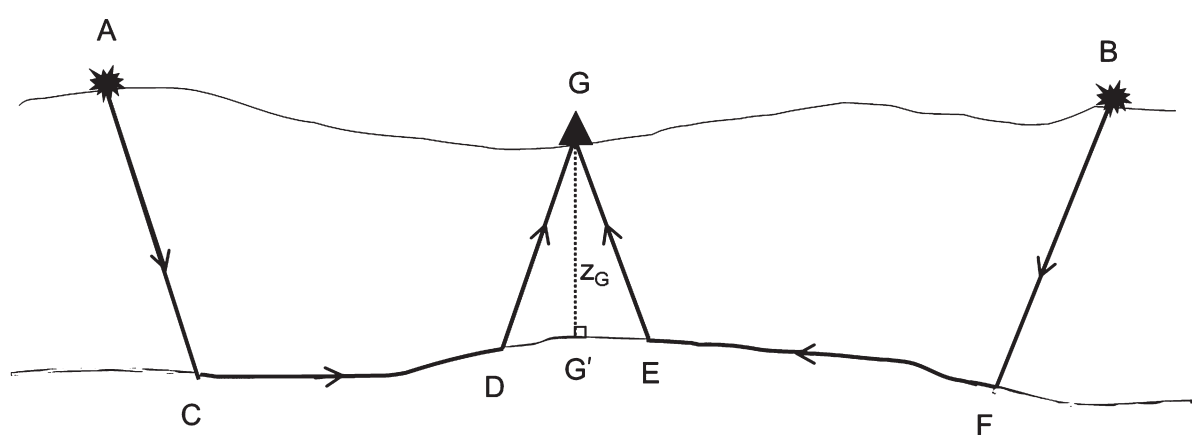

Fig. 5. Ray-path geometry for the reciprocal method. The forward refraction time $\left(t_{\mathrm{F}}\right)$ is the time to travel the path ACDG. The reverse time $\left(t_{\mathrm{R}}\right)$ is the time along BFEG. The reciprocal time $\left(T_{\mathrm{AB}}\right)$ is the time along ACFB. $G^{\prime}$ is a refractor point lying on the perpendicular from $\mathrm{G}$ (Modified from Hawkins, 1961). 
The refractor velocity $\left(V_{2 \mathrm{P}}\right)$ is then derived from this velocity function at any geophone via:

$$
V_{2 \mathrm{P}}=1 /\left(\text { slope of } \mathrm{t}_{V} \text { function }\right) .
$$

\section{Reciprocal method for PPS refraction}

To simplify the introduction to converted-wave concepts, we again restrict our explanations to the conventional reciprocal method. Figure 6 provides greater detail on the receiver region in Figure 5, and also adds the upgoing head waves for PPS refractions (dashed). We can derive an S-wave time-depth from the PPS refractions, again using equation 6 . Note that in this case the forward and reverse refraction times $\left(t_{\mathrm{F}}\right.$ and $\left.t_{R}\right)$ incorporate the converted-wave segments $\mathrm{D}^{\prime} \mathrm{G}$ and $\mathrm{E}^{\prime} \mathrm{G}$. Note that the reciprocal time $\left(T_{\mathrm{AB}}\right)$ is still the $\mathrm{P}$-wave shot-to-shot time. We can also derive a PPS velocity function using equation 8 , where again the quantities $t_{\mathrm{F}}$ and $t_{R}$ incorporate the converted-wave segments $\mathrm{D}^{\prime} \mathrm{G}$ and $\mathrm{E}^{\prime} \mathrm{G}$.

It is of interest to examine the relationship between the P-wave and $\mathrm{S}$-wave time-depths at a geophone, which we will denote as $t_{\mathrm{GP}}$ and $t_{\mathrm{GS}}$. Extending the $\mathrm{P}$-wave relationships given in equations 7 and 8 we have

$$
t_{\mathrm{GP}}=\mathrm{DG} / V_{1 \mathrm{P}}-\mathrm{DG}^{\prime} / V_{2 \mathrm{P}}=Z_{\mathrm{G}} \cos i_{\mathrm{P}} / V_{1 \mathrm{P}}
$$

and

$$
t_{\mathrm{GS}}=\mathrm{D}^{\prime} \mathrm{G} / V_{1 \mathrm{~S}}-\mathrm{D}^{\prime} \mathrm{G}^{\prime} / V_{2 \mathrm{P}}=Z_{\mathrm{G}} \cos i_{\mathrm{S}} / V_{1 \mathrm{~S}}
$$

where the path segments are as defined in Figure 6 , and $i_{\mathrm{P}}$ and $i_{\mathrm{S}}$ are the critical angles for $\mathrm{P}$ and $\mathrm{S}$, defined in equations 1 and 2 . We will use the term $\gamma^{*}$ to denote the ratio of the S-wave to P-wave time-depths at a geophone. Using equations 11 and 12, we obtain

$$
\gamma^{*}=t_{\mathrm{GS}} / t_{\mathrm{GP}}=\gamma\left(\frac{\cos i_{S}}{\cos i_{P}}\right)
$$

where $\gamma$ is the velocity ratio $V_{1 \mathrm{p}} / V_{1 \mathrm{~S}}$. Consideration of a range of reasonable values of $V_{1 \mathrm{P}}, V_{1 \mathrm{~S}}$ and $V_{2 \mathrm{P}}$, suggests that the trigonometric ratio in equation 13 is often close to one. Hence the time-depth ratio $\left(\gamma^{*}\right)$, which can be obtained directly from the refraction observations, often provides a good approximation to the $V_{\mathrm{P}} / V_{\mathrm{S}}$ velocity ratio $(\gamma)$ in the weathering zone. This parameter (or the related dynamic Poisson's ratio) is of interest in the context of engineering rock-strength determination.

Furthermore, with some knowledge of the surficial P-wave velocity $\left(V_{1 \mathrm{P}}\right)$ the measured $\gamma^{*}$ value can be further 'tuned' to provide a more accurate estimate of $\gamma$. Figure 7 indicates the percentage correction required to $\gamma^{*}$, for $V_{1 \mathrm{P}}$ values ranging from $500 \mathrm{~m} / \mathrm{s}$ to $3000 \mathrm{~m} / \mathrm{s}$. The dependence of the correction on the velocities can be clarified with reference to equation 13. First for high contrast situations $\left(V_{1}<<V_{2}\right)$ the cos terms in equation 13 approach one, as does their ratio. Hence, for smaller $V_{1}$ values, $\gamma^{*}$ approaches $\gamma$. Second, for $\gamma$ values close to one, $V_{1 \mathrm{~S}}$ approaches $V_{1 \mathrm{P}}$, and hence $\cos i_{\mathrm{S}}$ approaches $\cos i_{\mathrm{P}}$, for all values of $V_{1 \mathrm{P}}$. Hence the difference between $\gamma^{*}$ and $\gamma$ approaches zero as observed in Figure 7.

The process of tuning the observed $\gamma^{*}$ value to obtain an improved estimate of $\gamma$ ideally uses the known $V_{1 P}$ at a particular location. However, even if $V_{I P}$ is not accurately known, reasonable global adjustments can still be made. For example, if $V_{1 \mathrm{P}}$ were assumed to be generally in the region of $1000 \mathrm{~m} / \mathrm{s}$, then a reduction by $5 \%$ in $\gamma^{*}$ would provide an improved estimate of $\gamma$.

Meulenbroek and Hearn (2011) give a practical example of estimating near-surface $V_{\mathrm{P}} / V_{\mathrm{S}}$ ratios using the ratio of PPS and PPP time-depths.

\section{Ray-path considerations for dynamite recording Shot-depth corrections}

One of the more important commercial applications of seismic refraction is in deriving static corrections for reflection data. In this context it is interesting to consider practical implications of using subsurface explosive sources, which are often placed in compact material just below the weathering layer.

It is well known that for a shot buried at depth $h$ in the weathering layer (Figure $8 a$ ), the observed refraction times are reduced by an amount

$$
h \cos i_{\mathrm{P}} / V_{1 \mathrm{P}} .
$$

Thus when shots are in the weathering layer, recorded times must be increased by this amount, to effectively simulate a surface source. An approximation to this correction is to add the observed uphole time, if it is available.

An interesting simplification applies to the case where the shot lies just beneath the base of weathering (Figure $8 b$ ). Recall from equation 4 that for the case of a surface source the observed intercept time is $2 Z_{\mathrm{A}} \cos i_{\mathrm{P}} / V_{1 \mathrm{P}}$, where $Z_{\mathrm{A}}$ is the perpendicular depth to the refractor. If the shot is placed just below the refractor, then from equation 14 the refraction times are reduced by $Z_{\mathrm{A}} \cos$ $i_{\mathrm{P}} / V_{1 \mathrm{P}}$. That is for a source just below the refractor, the intercept time is halved (compared to a surface source) to become:

$$
T_{\mathrm{A}}=Z_{\mathrm{A}} \cos i_{\mathrm{P}} / V_{1 \mathrm{P}} .
$$

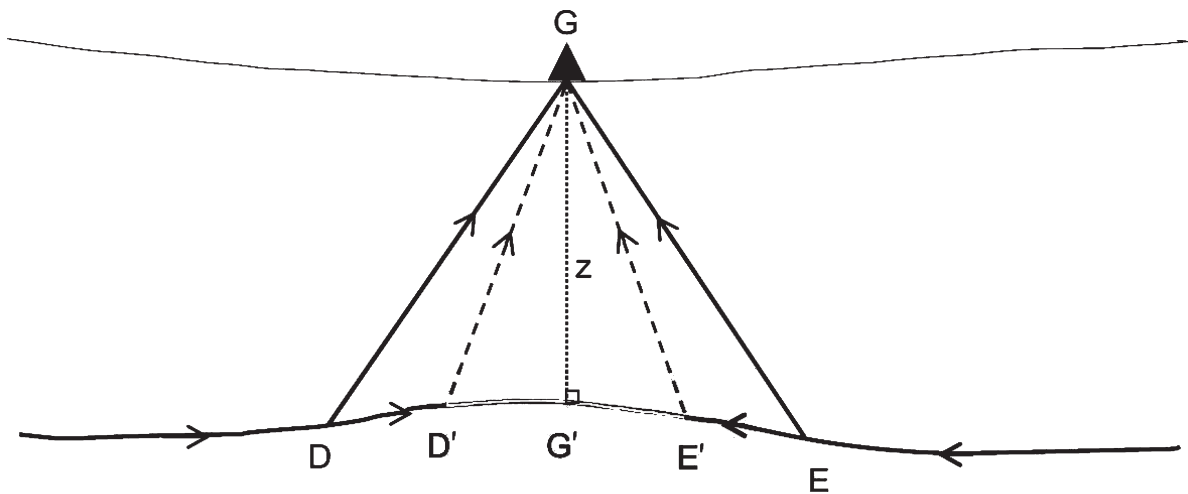

Fig. 6. Detail from the receiver region of Figure 5. The segments DG and EG are the PPP headwaves from forward and reverse shots. The segments $\mathrm{D}^{\prime} \mathrm{G}$ and $\mathrm{E}^{\prime} \mathrm{G}$ are the PPS headwaves from forward and reverse shots. 


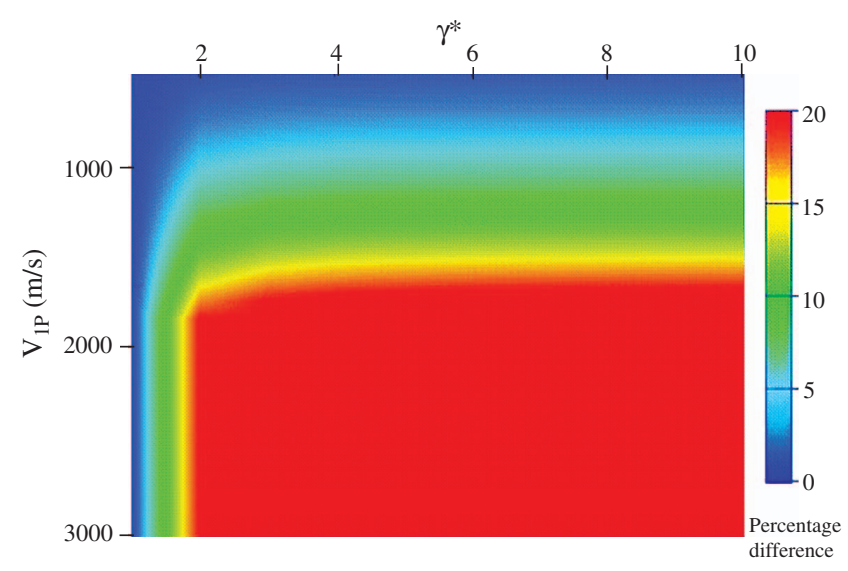

Fig. 7. Percentage difference between measured time-depth ratio, $\gamma^{*}$, and true $V_{\mathrm{P}} / V_{\mathrm{S}}$ ratio, $\gamma$, in the weathering layer, for a range of earth models $\left(V_{1 \mathrm{P}}\right.$ ranging from $500 \mathrm{~m} / \mathrm{s}$ to $3000 \mathrm{~m} / \mathrm{s}, \gamma^{*}$ ranging from 1 to $10, V_{2 \mathrm{P}}$ is set at a constant $3000 \mathrm{~m} / \mathrm{s}$ ). For plotting purposes, any difference above $20 \%$ is assigned an error $20 \%$ (Such $V_{1 \mathrm{P}}$ values are beyond those typically observed in real data examples).

Finally consider the case where the source is below the base of weathering by an amount $u$ (Figure $8 c$ ). Simple numerical experiments show that provided $u<<x$ (offset), the refraction times are approximately those for a source just below the refractor. That is, the result in equation 14 will hold for geophones that are a reasonable distance from the source. In practice this applies to geophones at offsets used in production refraction statics analysis. The following section explains how the relationship in equation 14 can be exploited to achieve an exact shotpoint correction, without the need for uphole times.

\section{Reciprocal times for shots in refractor}

To calculate time-depth (equation 6) and velocity function (equation 9) at a geophone we require an estimate of the PPP reciprocal time $\left(T_{\mathrm{AB}}\right)$. As noted above, this quantity is required for the reciprocal analysis of both PPP and PPS refraction data. For a surface source, $T_{\mathrm{AB}}$ can be obtained directly from the PPP traveltime plots, as was indicated in Figure 2. When the source is at depth in the weathering layer, shot-depth corrections (equation 14) can be applied to effectively impose a surface source.

Figure $9 a$ shows forward and reverse ray paths to a geophone for the case where the shots are situated in the refractor. (Although the refraction has only two segments when the source is in the refractor, we will use the term PPP refraction for consistency with the more general case.) Now, the required reciprocal time $\left(T_{\mathrm{AB}}\right)$ is the time along the path ADEB, fully within the refractor. This cannot be directly measured from travel-time plots. However, as shown in Figure $9 b$, an estimate can be made of the associated time $T_{\mathrm{AB}}{ }^{*}$, to a hypothetical Receiver $\mathrm{B}^{*}$ above the Shot B. Examination of Figure $9 a$, in the light of the preceding discussion of shot-depth corrections, indicates that the times $T_{\mathrm{AB}}$ and $T_{\mathrm{AB}} *$ are related via:

$$
T_{\mathrm{AB}}{ }^{*}=T_{\mathrm{AB}}+Z_{\mathrm{B}} \cos i_{\mathrm{P}} / V_{1 \mathrm{P}},
$$

where $Z_{B}$ is the depth to the refractor at Shot B. However, as discussed above (equation 15) the rightmost term is just the intercept time for the refraction branch at Shot B, which is directly measurable on the travel-time plot $\left(T_{\mathrm{B}}\right.$ on Figure $\left.9 b\right)$. Hence the required reciprocal time can in practice be obtained as

$$
T_{\mathrm{AB}}=T_{\mathrm{AB}}^{*}-T_{\mathrm{B}}
$$

where both terms on the right-hand side can be measured from the travel time plot (Figure 9b).

In a similar fashion, the reciprocal time $T_{\mathrm{BA}}$ can be estimated via:

$$
T_{\mathrm{BA}}=T_{\mathrm{BA}}^{*}-T_{\mathrm{A}}
$$

where the terms $T_{\mathrm{BA}}{ }^{*}$ and $T_{\mathrm{A}}$ are directly measurable from the travel-time plot at Shot A (Figure $9 b$ ).

Finally, note that the measured times $T_{\mathrm{AB}} *$ and $T_{\mathrm{BA}}$ * will generally not be equal, because they relate to different paths. However, the corrected times $T_{\mathrm{AB}}$ and $T_{\mathrm{BA}}$ should, in theory, satisfy reciprocity.

\section{Relationship between time-depth and delay-time}

Where reversed refraction arrivals are available, the reciprocal approach has the advantage of elegantly handling ambiguities between refractor velocity and dip. In practice, there may be zones where reversed data may not be available due to poor signal-tonoise on the refracted data. This situation is more likely for PPS refractions, since these are not first arrivals. Where only singlesided arrivals are available, the delay-time approach (Gardner, 1939) provides a workable alternative to the reciprocal method. As shown below, a particularly elegant integration of time-depth and delay-time results is possible for the case where the source lies in the refractor.

We will introduce the delay-time concept for the case of a surface source. As illustrated in Figure 10, surface points are (a)

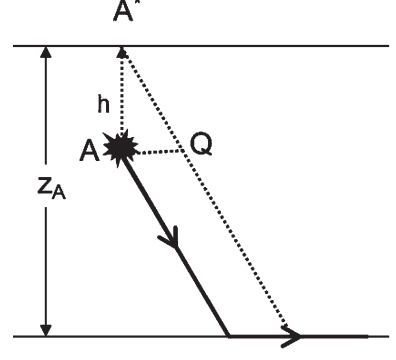

(b)

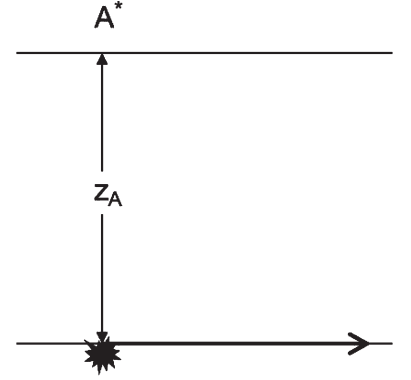

(c)

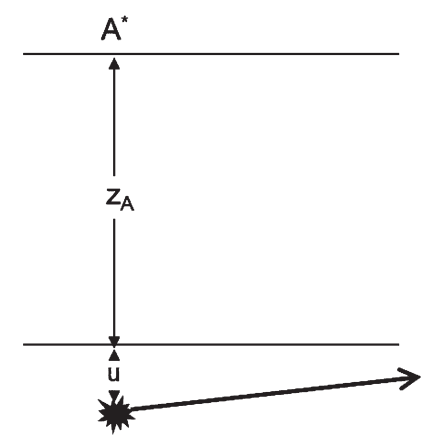

Fig. 8. Shot-depth corrections. (a) Shot within weathering layer at depth $h$ below surface: Correction to move shot at depth (A) to the surface (A*) is $\mathrm{A} * \mathrm{Q} / V_{1 \mathrm{P}}-\mathrm{AQ} / V_{2 \mathrm{P}}$, which reduces to $h \cos i_{\mathrm{P}} / V_{1 \mathrm{P}}$. (b) Shot just below weathering layer: Correction is $Z_{\mathrm{A}} \cos i_{\mathrm{P}} / V_{1 \mathrm{P}}$. (c) Shot below weathering: if $u<<x$ (offset) the refraction time is approximately equal to that for the situation in $(b)$. 

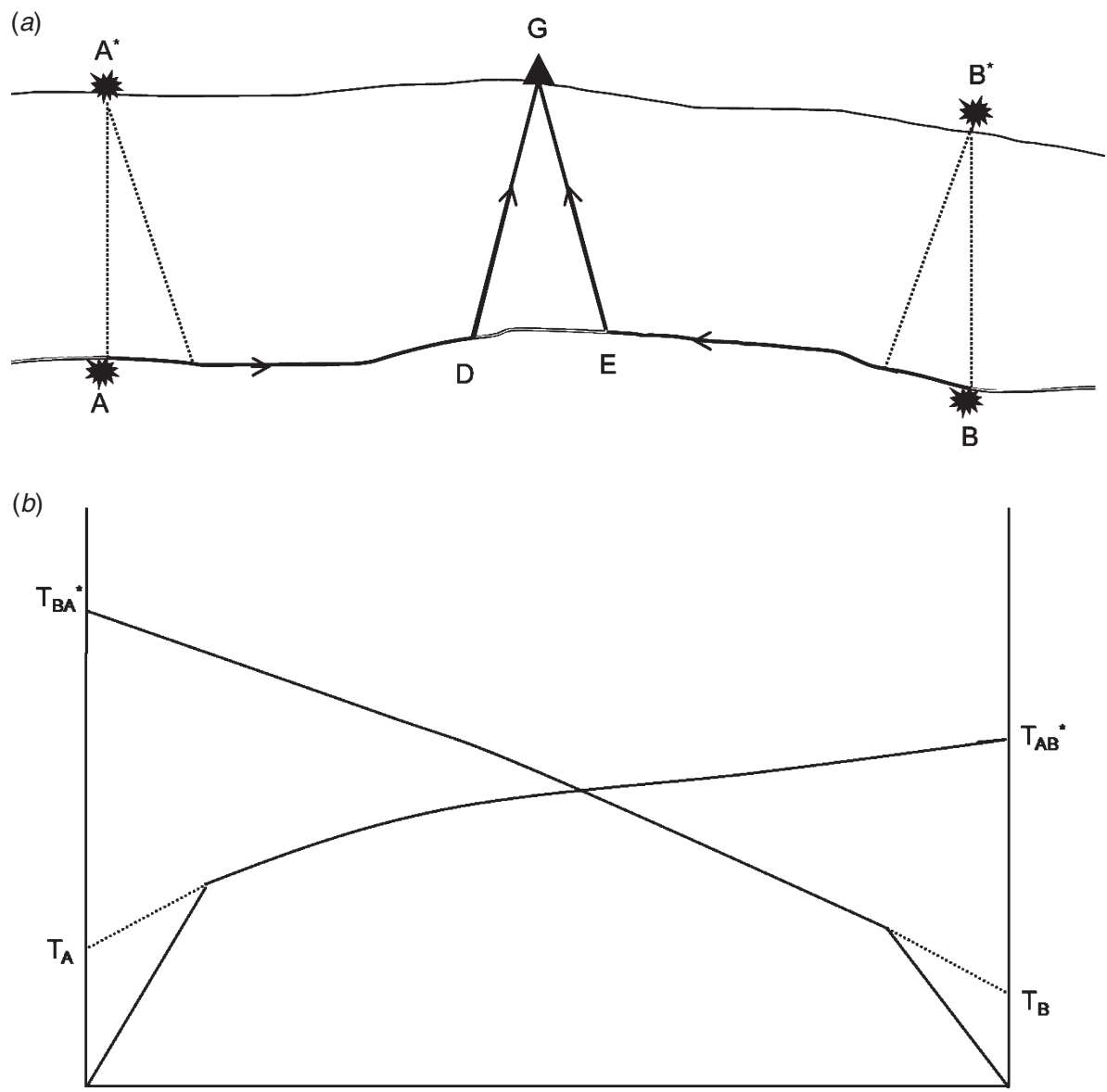

Fig. 9. (a) Reciprocal method geometry for shots within the refractor. The required reciprocal time $\left(T_{\mathrm{AB}}\right)$ is not directly measurable from the travel-time plots. However, the associated times $T_{\mathrm{AB}} *$ and $T_{\mathrm{BA}}{ }^{*}$ are measurable. (b) Travel-time plots corresponding to the situation in $(a)$. The required reciprocal time $\left(T_{\mathrm{AB}}\right)$ can be derived from parameters measured from the plot, $T_{\mathrm{AB}}=T_{\mathrm{AB}} *-T_{\mathrm{B}}=T_{\mathrm{BA}} *-T_{\mathrm{A}}$.

associated with refractor points vertically below them. This is a subtle difference to the slope-intercept and the time-depth methods which utilise refractor points lying on perpendiculars (e.g. $G^{\prime}$ in Figure 5). Following Gardner (1939), and referring to Figure 10, the total PPP refraction time from a shot at A to a geophone at $\mathrm{G}\left(t_{\mathrm{F}}\right)$ is adjusted to yield a reduced refraction time.

$$
b_{\mathrm{P}}=t_{\mathrm{F}}-\mathrm{AG} / V_{2 \mathrm{P}}
$$

The terms on the right hand side can be broken down into individual segments, such that:

$$
b_{\mathrm{P}}=\left[\mathrm{AC} / V_{1 \mathrm{P}}-\mathrm{A}^{\prime} \mathrm{C} / V_{2 \mathrm{P}}\right]+\left[\mathrm{DG} / V_{1 \mathrm{P}}-\mathrm{DG}^{\prime} / V_{2 \mathrm{P}}\right] \text {, }
$$

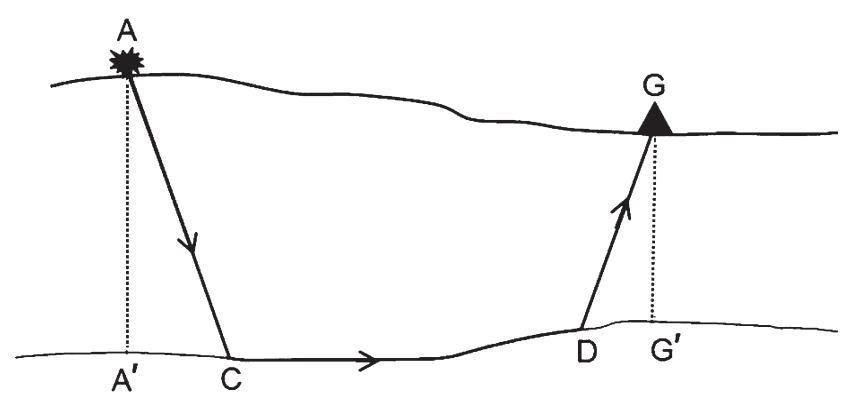

Fig. 10. Ray-path geometry for the delay-time method, which is based on a single-sided refraction arrivals. The refraction time $\left(t_{\mathrm{F}}\right)$ is the time to travel the path ACDG. The delay-time definition utilises refractor points $A^{\prime}$ and $G^{\prime}$, which are vertically below A and G.
Gardner (1939) refers to the terms in square brackets as the delay-times at source (A) and receiver $(\mathrm{G})$. Comparison with equation 11 indicates that Gardner's delay-time is approximately equal to the time-depth from reciprocal analysis. The approximation relates to the different definitions of the refractor points $\left(\mathrm{A}^{\prime}, \mathrm{G}^{\prime}\right)$ as noted above. When the local dip is not severe, this difference in definition is not significant. The approximation is generally acceptable in practical situations. Note that the reduced time $\left(b_{\mathrm{P}}\right)$ is approximately the sum of time-depths at source and receiver.

$$
b_{\mathrm{P}} \sim t_{\mathrm{AP}}+t_{\mathrm{GP}}
$$

The idea can immediately be extended to PPS refraction. The observed PPS refraction time can be reduced to a quantity $b_{\mathrm{S}}$, again using the estimated $\mathrm{P}$-wave refractor velocity, as in equation 19. Now, however, the receiver delay-time component relates to the $\mathrm{S}$ head wave (Figure 6), and is approximately equal to the S-wave time-depth given in equation 12 . That is, the reduced PPS refraction time is, approximately, the sum of the $\mathrm{P}$-wave time-depth at the source and the S-wave time-depth at the receiver.

$$
b_{\mathrm{S}} \sim t_{\mathrm{AP}}+t_{\mathrm{GS}}
$$

Of practical interest, note that when the source is in the refractor, the source delay-time $\left(t_{\mathrm{AP}}\right)$ is zero. Hence, the reduced PPP and PPS refraction times are simply:

$$
b_{\mathrm{P}} \sim t_{\mathrm{GP}} \text {, and }
$$




$$
b_{\mathrm{S}} \sim t_{\mathrm{GS}} .
$$

That is, the reduced refraction time derived from single-sided refraction data can be directly compared to the time-depth derived from reversed data. This approach of integrating time-depth and delay-time results is demonstrated in Meulenbroek and Hearn (2011).

Finally we include a note on the practical viability of the delaytime approach. Palmer(1986) suggests that the major drawback of the delay-time approach is that it has no inbuilt mechanism for estimating refractor velocity. This leads to inaccuracy in the reduction of times using equation 19 . These drawbacks are the direct result of analysing only single-sided data, where there must always be ambiguity between refractor velocity and dip.

These reservations apply to conventional PPP analysis. In our work, however, we typically restrict our use of delay-times to the analysis of PPS data, in circumstances where we cannot pick reversed PPS arrivals. In this situation, the refractor velocity $\left(V_{2 \mathrm{P}}\right)$ profile has typically already been calculated from PPP refractions, using the reciprocal method. Hence the delay-time analysis of PPS arrivals (using equation 19) can be considered more reliable. Where the PPP reciprocal analysis indicates that the refractor velocity is changing laterally, it is appropriate that the delay-time reduction in equation 19 be modified to:

$$
b=t_{\mathrm{F}}-\Sigma_{k}\left(\delta x_{k} / V_{2 \mathrm{P} k}\right)
$$

where the offset distance AG has been broken into segments $\delta x_{k}$ with $V_{2 \mathrm{P} k}$ being the refractor $\mathrm{P}$ velocity in segment $k$.

\section{Conclusion}

The objective of this paper has been to outline basic ray-path concepts required for analysis of converted-wave (PPS) refractions, for the primary purpose of converted-wave statics analysis. We have shown that this is a relatively straightforward extension of conventional PPP refraction theory. We have outlined several simplifications which arise when the source is situated in the refractor, as is common in dynamite shooting. Reciprocal times can be easily corrected for shot depth using measured intercept times. In addition, in zones where reversed data are not available, delay-times can be readily integrated with the time-depths obtained from reversed data.

This discussion has been restricted to the simple overburdenbedrock model, which is widely used in refraction statics analysis. For clarity, we have restricted this discussion to the conventional form of the reciprocal method, although the results are easily applied to other related algorithms.
The concepts introduced here provide the basis for a practical evaluation of converted-wave refraction, which is presented in a companion paper (Meulenbroek and Hearn, 2011).

\section{Acknowledgements}

We thank Derecke Palmer and two anonymous reviewers of this manuscript.

\section{References}

Edge, A. B., and Laby, T.H., 1931, The Principles and Practice of Geophysical Prospecting: being the report of the Imperial Geophysical Experimental Survey: University Press.

Gardner, L. W., 1939, An areal plan of mapping subsurface structure by refraction shooting: Geophysics, 4, 247-259. doi:10.1190/1.1440501

Hagedoorn, J. G., 1959, The plus-minus method of interpreting seismic refraction lines: Geophysical Prospecting, 6, 285-314.

Hagiwara, T., and Omote, S., 1939, Land creep at Mt Tyausu - Yama (Determination of slip plane by seismic prospecting): Tokyo University Earthquake Research Institute Bulletin, 17, 118-137.

Hawkins, L. V., 1961, The reciprocal method of routine shallow seismic refraction investigations: Geophysics, 26, 806-819. doi:10.1190/1.143 8961

Knox, W. A., 1967, Multilayer near-surface refraction computations, in A.W. Musgrave, ed., Seismic refraction prospecting: Society of Exploration Geophysicists, 197-216.

Li, X. Y., Dai, H., Mueller, M., and Barkved, O., 2001, Compensating for the effects of gas clouds on C-wave imaging: a case study from Valhall: The Leading Edge, 20, 1022-1028. doi:10.1190/1.1487307

Meulenbroek, A., and Hearn, S., 2011, Analysis of converted-refractions for shear statics and near-surface characterisation: Exploration Geophysics, 42, 147-154. doi:10.1071/EG10031

Musgrave, A. W., 1967, ed., Seismic refraction prospecting: Society of Exploration Geophysicists.

Nettleton, L. L., 1940, Geophysical prospecting for oil: McGraw Hill.

Palmer, D., 1981, An introduction to the generalized reciprocal method of seismic refraction interpretation: Geophysics, 46, 1508-1518. doi:10.1190/1.1441157

Palmer, D., 1986, Refraction seismics - The lateral resolution of structure and seismic velocity: Geophysical Press.

Thompson, L., 1999, Converted-wave reflection seismology over inhomogeneous, anisotropic media: Geophysics, 64, 678-690. doi:10.1190/1.1444577

Manuscript received 27 October 2010; accepted 26 May 2011. 